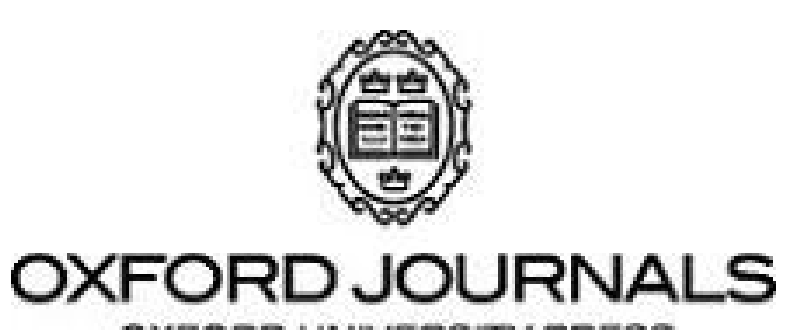

OXFORD UNIVERSITY PRESS

The British Society for the Philosophy of Science

Induction and Scientific Realism: Einstein versus van Fraassen: Part Two: Aim-Oriented Empiricism and Scientific Essentialism

Author(s): Nicholas Maxwell

Source: The British Journal for the Philosophy of Science, Vol. 44, No. 1 (Mar., 1993), pp. 81101

Published by: Oxford University Press on behalf of The British Society for the Philosophy of Science

Stable URL: http://www.jstor.org/stable/687851

Accessed: $16 / 03 / 2011$ 19:40

Your use of the JSTOR archive indicates your acceptance of JSTOR's Terms and Conditions of Use, available at http://www.jstor.org/page/info/about/policies/terms.jsp. JSTOR's Terms and Conditions of Use provides, in part, that unless you have obtained prior permission, you may not download an entire issue of a journal or multiple copies of articles, and you may use content in the JSTOR archive only for your personal, non-commercial use.

Please contact the publisher regarding any further use of this work. Publisher contact information may be obtained at http://www.jstor.org/action/showPublisher?publisherCode=oup.

Each copy of any part of a JSTOR transmission must contain the same copyright notice that appears on the screen or printed page of such transmission.

JSTOR is a not-for-profit service that helps scholars, researchers, and students discover, use, and build upon a wide range of content in a trusted digital archive. We use information technology and tools to increase productivity and facilitate new forms of scholarship. For more information about JSTOR, please contact support@jstor.org. 


\title{
Part Two: Aim-oriented Empiricism and Scientific Essentialism
}

\author{
NICHOLAS MAXWELL
}

In this second part of the present three-part paper, my concern is to show that aim-oriented empiricism provides decisive grounds for accepting scientific realism, and rejecting van Fraassen's anti-realist constructive empiricism (van Fraassen [1980, 1985]). But the argument goes further than this. For I shall argue that physicalism is a central part of our current (conjectural) scientific knowledge. And furthermore, I shall argue not just for scientific realism but, in addition, for conjectural (scientific) essentialism - the doctrine that we need to interpret (appropriate) fundamental dynamic physical theories as attributing necessitating physical properties to postulated physical entities such as particles and fields. During the course of the argument I will show how, within the framework of aim-oriented empiricism, rather straightforward solutions can be given to the problems of how, given that science advances from one false theory to another, (1) it can make sense to speak of scientific progress (the problem of verisimilitude), and (2) how it can be valid to interpret current physical theories as providing us with genuine knowledge of unobservable physical entities. It will emerge, furthermore, that the way in which science advances, from one false theory to another, does not undermine but, if anything, actually supports convergent realism. This way for science to make progress is just what we should expect if physicalism is true!

I develop my case in support of conjectural (scientific) essentialism, and against van Fraassen's anti-realist constructive empiricism in the following six points.

1. Constructive empiricism, the instrumentalist conception of science van Fraassen defends, is a version of standard empiricism. We have seen, however, in part one of this paper, that in order to solve the problem of induction-in order to construe science as a rational, intellectually rigorous enterprise-it is essential to adopt aim-oriented empiricism, and reject all versions of standard empiricism. This requires that we reject constructive empiricism. 
2. Van Fraassen's arguments against the kind of scientific realism to be advocated here all devolve from the crucial assumption that the only valid basis for scientific knowledge is experience of the observable. (It is this which leads van Fraassen to conclude that there can be no valid basis for scientific knowledge of the unobservable.) We have seen, however, in part one of this paper, that this crucial assumption must be rejected. Experience of the observable cannot provide a valid basis for rejecting the aberrant predictions of empirically successful aberrant theories and accepting instead, as knowledge (or belief) the predictions of less empirically successful non-aberrant, explanatory theories. Explanatoriness is an indispensable and legitimate consideration to be taken into account in judging what constitutes scientific knowledge, in addition to experience of the observable. An essential premise of van Fraassen's arguments against the kind of scientific realism advocated here must, in other words, be rejected, the result being that these arguments against scientific realism all collapse.

3. In sharp contrast to what van Fraassen claims, scientific realism is implicit in the metaphysical presuppositions of contemporary scientific knowledge, and implicit in a fundamental aim of science. There are three points to be made.

(i) Knowledge about what is unobservable is implicit in physicalism which is in turn implicit in current (conjectural) scientific knowledge. The arguments of part one of this paper established that in order to make rational sense of science we must construe (conjectural) scientific knowledge to include the thesis that the universe is comprehensible in some way or other and, more specifically, that it is comprehensible in the kind of way specified by physicalism. But this requires that scientific knowledge is in part knowledge of the unobservable, since what physicalism asserts to exist, the invariant $\mathrm{U}$, and the variable $\mathrm{V}$ will, throughout most of space and time, be unobservable. (In this respect $U$ and $V$ will be rather like what is invariant and variable in gravitational fields, electromagnetic fields, curved space-time, quarks, superstrings, and early states of the universe which preclude the existence of observers. Only special physical states of affairs, special values of $V$, will constitute such observable entities as stones, birds, stars.)

(ii) The idea of a true, realistically interpreted physical theory which is in part about what is unobservable is implicit in current scientific knowledge. Physicalism, a part of scientific knowledge, asserts that the world is such that it is in principle possible to formulate some true, unified, comprehensive physical theory $\mathrm{T}$, which specifies precisely the nature of $U$ and $V$, and which thus specifies how $U$ determines (perhaps probabilistically) the way $\mathrm{V}$ varies. This as-yet-to-bediscovered theory $\mathrm{T}$, the idea of which is implicit in physicalism, would clearly be in part about the unobservable. It is in this sense, then, that the idea of a 
realistically interpreted physical theory about the unobservable is implicit in current scientific knowledge.

(iii) A proper basic aim of science is to acquire (or improve) knowledge of the unobservable. The basic aim of theoretical physics-and thus a basic aim of the whole of science-is to discover T (as just characterized); it is, in other words, to transform physicalism into a true, precise, unified, comprehensive, corroborated physical theory, which specifies precisely the nature of $\mathrm{U}$ and $\mathrm{V}$. In this way, scientific realism is built into a basic aim of science, into the whole research programme of theoretical physics.

What these three points bring out is that in order to make rational sense of science (in accordance with aim-oriented empiricism) we must see science as already including some (admittedly imprecise) knowledge of the unobservable, and being committed to improving this knowledge of the unobservable.

4. As science advances and explanatory, empirically successful theories are superseded (from the standpoint of fundamental theoretical physics) by theories of greater explanatory power, empirical content and empirical success - theories which explain the (partial) empirical successes of the earlier, superseded theories - it almost always happens that the new theories correct the old theories, and reveal the old theories to be, strictly speaking, false. Thus Newtonian theory shows that Kepler's laws cannot be precisely correct. Einstein's theory of general relativity shows that Newtonian theory cannot be precisely correct. Quantum theory corrects all the theories of classical physics. Quantum electrodynamics corrects non-relativistic quantum theory, and the Salam-Weinberg electroweak theory corrects quantum electrodynamics. On the other hand, the electroweak theory and the more recent theory of quantum chromodynamics cannot be quite right either, granted that general relativity is at least approximately correct, since these theories presuppose flat space-time and general relativity tells us that space-time is curved by the presence of energy. It seems, then, that all physical theories so far proposed, from Kepler and Newton to Salam and Weinberg are, strictly speaking, false.

That physics does advance in this way, from one false theory to another, may be thought to render physicalism (and hence aim-oriented empiricism) highly implausible. ${ }^{1}$ But this is not so. Granted the truth of physicalism, it is actually to be expected that physics advances from one false theory to another until the true theory of everything is achieved. If a theory, $\mathrm{T}_{0}$, is precisely true throughout some restricted domain of phenomena $\mathrm{D}$ then, granted physicalism, $T_{0}$ must specify precisely $U$, and the way $U$ determines change in $V$,

${ }^{1}$ Both Laudan [1980] and Newton-Smith [1981] argue that the fact that science advances from one false theory to another poses a severe problem for 'convergent realism'- the doctrine that science is progressively improving our knowledge of unobservable physical reality. 
throughout D. But according to physicalism, U exists unchanged throughout all phenomena; furthermore, its nature or structure is given by the basic form of the theory which correctly specifies how $\mathrm{U}$ determines change in $\mathrm{V}$. This means that if $\mathrm{T}_{0}$ is correct in $\mathrm{D}$, it ought to be easily possible to extend the interpretation and application of $\mathrm{T}_{0}$ to all phenomena, keeping the form of $\mathrm{T}_{0}$ unchanged, in this way arriving at the ultimately true theory of everything, $\mathrm{T}$. Conversely, if $\mathrm{T}_{0}$ cannot be extended in this way to apply correctly to all phenomena, then $\mathrm{T}_{0}$ cannot be precisely true within $\mathrm{D}$ : $\mathrm{T}_{0}$ must be false. In brief, physicalism implies that a physical theory can only be precisely true of anything if it is (capable of being) precisely true of everything. Granted, then, that progress in physics involves the development of theories which meet with increasingly accurate empirical success throughout ever increasing domains of phenomena, but which are not empirically successful for all phenomena, it is inevitable (granted physicalism) that physics will progress by the development of theories that are all false throughout their domain of application until the ultimate, unified theory of everything is attained (which will be precisely true about everything). Since physicalism predicts that physics progresses in this way, the fact that physics has so far thus progressed can only count in favour of physicalism: it cannot count against physicalism (as some appear to suppose).

There is just one conceivable exception to this argument. It is possible that the form of $\mathrm{T}$ (or the nature of $\mathrm{U}$ ) might be such that $\mathrm{T}$ reduces to an especially simple form for an especially simple or appropriately symmetric kind of system. (Two spherical bodies of equal mass rotating about the point midway between them exemplify a law much simpler in form than Newtonian theory.) In having only what remains of $\mathrm{T}$ when it has been reduced to just such an especially simple form for some simple or symmetric system, one would have a true theory, but a theory not easily extendable to recover T. However, even if such a simplified version of $\mathrm{T}$ were to be formulated, it is most unlikely, before the discovery of $\mathrm{T}$, that it would be correctly interpreted to apply only to the appropriately symmetric kind of system. (One would need to have $\mathrm{T}$ in order to know how to specify correctly systems to which the simplified version of $\mathrm{T}$ applies precisely.) Interpreted to apply to a broader range of systems, the simplified version of $\mathrm{T}$ will not be precisely true.

That physics has so far progressed in the characteristic way in which it has, from one false theory to another, if anything supports, and does not count against, aim-oriented empiricism in so far as aim-oriented empiricism is taken to assert no more than that physicalism, and therefore realism, is implicit in the basic aim of theoretical physics. A difficulty does, however, arise if aim-oriented empiricism is taken to assert, in addition, that it is legitimate to interpret existing accepted physical theories realistically, as providing us with knowledge about the unobservable entities the theories ostensibly postulate. How can this be if all these theories are false? For if Newtonian theory, classical electrodynamics, general relativity and quantum field theory are all false, does not this 
mean that the physical entities postulated by these theories-classical particles and fields, curved space-time, quantum fields-do not exist? If any of these entities do exist, with precisely the properties attributed to them by the corresponding theory, would not this mean that this theory is true? What then becomes of scientific realism as far as existing experimentally confirmed but false physical theories are concerned?

One might attempt to solve this problem by exploiting Saul Kripke's point that we can successfully refer to an object by means of false statements: see Kripke ([1980], pp. 83-93). Such an attempt would maintain that existing physical theories can (and do) refer to unobservable, theoretical entities even though these theories are, strictly speaking, false.

I cannot avail myself of this approach to the problem, however, for two reasons. First, in my view Kripke's account of reference is only partly correct. Kripke is right to point out that when we identify some object by means of a definite description (as when we identify Aristotle as the last great Greek philosopher), the properties which the description attributes to the object need not be essential ones-indeed they do not even need to be properties of the object at all. Kripke is wrong, however, to maintain that identity statements formulated in terms of rigid designators, are, if true, necessarily true. In these circumstances, calling upon even a part of Kripke's theory of reference would only lead to confusion. But second, even if Kripke's theory of reference were entirely correct, it could not be used to solve our problem. For in order to solve this problem, we need to be able to show that existing physical theories, even though false, can nevertheless provide us with (conjectural) scientific knowledge about entities at least approximately like the unobservable entities postulated by the theories in question. Kripke's account of reference is of no help here (the import of this account being, in a sense, that knowledge is not required for reference).

The way I propose to solve the problem is to establish the following two points. First, granted the truth of physicalism, explanatory theories that have met with great empirical success throughout some domain of phenomena (like Newtonian theory, general relativity or quantum theory) are not just false: such theories are approximately true, or lead to theories of a kind that are absolutely true, within some restricted domain of phenomena. Second, even if entities precisely like those postulated by these empirically successful but false theories do not exist, nevertheless the approximate truth of these theories within a restricted domain of phenomena ensures that (unobservable) entities approximately like those postulated by the theories do exist. We can, therefore, hold that existing physical theories provide us with knowledge of unobservable entities, even though these theories are, strictly speaking, false. Aim-oriented empiricism not only holds that scientific realism is implicit in physicalism and the ultimate aim of theoretical physics (point 3 above): it also provides a basis 
for holding that existing scientific theories, when interpreted realistically in the qualified sense indicated, may well be true, and in any case provide us with (conjectural) scientific knowledge of unobservable entities.

I take these two points in turn.

(i) When an explanatory, empirically successful theory $T_{0}$ is superseded by a theory $\mathrm{T}_{1}$, which has greater explanatory power, empirical content and empirical success, and which explains the (partial) empirical success of $\mathrm{T}_{0}$, it is not the case that $T_{1}$, granted for the moment that it is true, merely shows $T_{0}$ to be false; it also shows that $\mathrm{T}_{0}$ is approximately true. In an important sense, $\mathrm{T}_{0}$ can be 'derived' from $\mathrm{T}_{1}$, and this 'derivation' shows how we can specify a restricted domain of phenomena within which $\mathrm{T}_{0}$ will be accurate to some given degree (the more accurate the smaller the domain). Thus, for example, Kepler's laws (KL) can be 'derived' from Newtonian theory (NT) in the following three steps. First, NT is restricted to N body systems interacting by gravitation alone within some definite volume, no two bodies being closer than some given distance r. Second, keeping the mass of one object constant, we consider the paths followed by the other bodies as their masses tend to zero. According to NT, in the limit, these paths are precisely those specified by KL for planets. In this way we recover the form of KL from NT. Third, we reinterpret this 'derived' version of KL so that it is now taken to apply to systems like that of our solar system. (It is of course this third step of reinterpretation that introduces error: mutual gravitational attraction between planets, and between planets and the sun, ensure that the paths of planets, with masses greater than zero, must diverge, however slightly, from precise Keplerian orbits.)

That KL can be 'derived' from NT in this way, by means of these three steps, enables us to make sense of the idea that KL is approximately true, granted the truth of NT. More to the point, it enables us to define an approximate version of $\mathrm{KL}, \mathrm{KL}^{*}$ say, which is precisely and absolutely true granted the truth of NT. (Even though KL is logically incompatible with NT, KL* is logically entailed by NT.)

We consider only Newtonian systems restricted by the first of the above three steps. Given the initial state of any such system at time $t_{0}$, then at any time $t>t_{0}$, the state of the system as predicted by NT will diverge somewhat from the state predicted by KL. Let this divergence $d$ at any instant $t$ be the maximum distance between what NT predicts for any object of the system, and what KL predicts for that object. (We can put the origin of our reference frame at the centre of mass of the whole system.) There are now two possibilities to consider, the time-independent case, and the time-dependent case.

The time-independent case. We suppose that, for appropriately restricted systems, NT tends to KL in a time-independent way, as the masses of the planet-like objects tend to zero. That is, given $\delta>0$, there exists $\delta \mathrm{m}>0$, such 
that, for all systems of the specified type with masses of each planet $<\delta \mathrm{m}$, the predictions of NT diverge from those of KL by an amount $\mathrm{d} \leqslant \delta$ for all subsequent times.

Given this, we can formulate $\mathrm{KL}^{*}$ to assert: 'For systems appropriately restricted, and further restricted by the requirement that the mass of each planet-like object $<\delta \mathrm{m}$, the paths of the planets diverge from Keplerian orbits by no more than $\delta$.' This, we see, is logically entailed by NT.

We may have set things up so that $\delta \mathrm{m}$ is very much smaller than the masses of actual planets. By demanding that $r$, the minimum distance between objects, is sufficiently increased, we can always arrange for $\delta \mathrm{m}$ to be increased by as much as we please. In order to ensure that the planet-like objects do not diverge from KL orbits by more than the given amount $\delta$, we must ensure that these objects do not exert gravitational forces on each other, and on the sunlike object, by more than a certain amount. Given $\mathrm{F}=\mathrm{Gm}_{1} \mathrm{~m}_{2} / \mathrm{d}^{2}$, we can arrange for this, either by having a sufficiently small $\delta \mathrm{m}$ or, where $\delta \mathrm{m}$ is large, by having a correspondingly large mass for the sun-like object, and ensuring that the minimum distances between objects is sufficiently large.

The time-dependent case. It may be that NT tends to KL in only the following time-dependent sense: Given $\delta>0$, and given $\mathrm{T}>\mathrm{t}_{0}$, there exists $\delta \mathrm{m}>0$ such that, for all systems of the specified type with masses of each planet $<\delta \mathrm{m}$, the predictions of NT diverge from those of KL by an amount $\mathrm{d} \leqslant \delta$ for all times $t$ for which $\mathrm{t}_{0} \leqslant \mathrm{t}<\mathrm{T}$. In this case $\mathrm{KL}^{*}$ must be formulated in a time-restricted manner, if $\mathrm{KL}^{*}$ is to be logically entailed by NT.

The points just made concerning NT, KL and $\mathrm{KL}^{*}$ can be made, I suggest, quite generally. Whenever (within the physicalist programme of physics) an explanatory, empirically successful theory $T_{0}$ is superseded by a theory $T_{1}$ (so that $T_{1}$ has greater explanatory power, empirical content and success than $T_{0}$, and is able to explain the partial empirical success of $T_{0}$, then $T_{0}$ can be 'derived' from $\mathrm{T}_{1}$ by means of steps analogous to the above three steps (or some repeated sequence of such steps). Furthermore, this enables us to define $T_{0}^{*}$, an approximate version of $T_{0}$, which is logically entailed by $T_{1}$, even though $T_{0}$ is incompatible with $\mathrm{T}_{1}$.

We have good reasons, I believe, to hold that these general points are true. Whenever a new theory, $\mathrm{T}_{1}$, is proposed in physics, it is standard procedure to show how relevant, pre-existing, more limited laws and theories, $\mathrm{T}_{0}$, can be 'derived' as approximations from the new theory. Strictly speaking, all such 'approximate derivations' (as they may be called) of $\mathrm{T}_{0}$ from $\mathrm{T}_{1}$ are invalid (because $T_{0}$ is incompatible with $T_{1}$ ). What entitles us to regard such 'derivations' as valid is that it is always an easy (if tedious) matter to reformulate the derivation so that $\mathrm{T}_{1}$ logically implies some $\mathrm{T}_{0}^{*}$ (some approximate version of $\mathrm{T}_{0}$ ). In practice, physicists may not bother to transform invalid 'approximate derivations' into valid derivations of approximate laws 
and theories in this way, not because this is too difficult, but because it is too pedantic. We have every reason, in short, to hold that the above remarks concerning NT, KL and $\mathrm{KL}^{*}$ can be generalized to apply to all cases where one theory, $\mathrm{T}_{0}$, can be 'approximately derived', as a limiting case, from another theory, $\mathrm{T}_{1}$.

It is worth noting just how ubiquitous 'approximate derivations' of the above type are in physics. When empirical predictions are derived from a physical theory approximations are very frequently made during the course of the derivation. Higher-order terms in some expansion are set to zero; complex expressions reduce to simple ones as a result of the neglect of effects deemed to be sufficiently minute. All such 'approximate derivations', to be found everywhere in physics, are logically invalid in just the same way in which the derivation of KL from NT is invalid. It is legitimate to regard such 'derivations' as valid in so far as it is an easy, if pedantic, matter to turn them into valid derivations by replacing the precise conclusion with an approximate one. None of this ought to seem problematic to anyone with any firsthand familiarity with physics. If it has seemed problematic to some philosophers, this can only be because they have put too much emphasis on understanding physical theories as propositions, and not enough on understanding scientiic practice.

The points just made make it possible to provide a straightforward solution to the problem of what it can mean to speak of scientific progress if science proceeds by moving from one false theory to another-the problem which led Popper to invent the notion of verisimilitude (Popper [1963], Ch. 10 and pp. 391-7), which in turn led to the problem of verisimilitude, the problem of what it can mean to say, of two false theories, that one has more verisimilitude (or is closer to the truth) than the other (Miller [1974], Tichý [1974]). Given a succession of false physical theories, $T_{0}, T_{1}, T_{2}, \ldots T_{n}$, 'progress towards the truth', within the framework of aim-oriented empiricism, can be defined in two ways. First, there is what may be called 'theoretical progress'-progress in theoretical understanding. According to aim-oriented empiricism, we are entitled to hold that the universe is physicalistically comprehensible. Let $\mathrm{T}$ be the comprehensive, unified, true 'theory of everything'. Suppose $\mathrm{T}$ is such that $T_{n}$ can be 'derived' from $T, T_{n-1}$ from $T_{n}$ and $\ldots T_{0}$ from $T_{1}$. Suppose further that none of these 'derivations' can be reversed: $T_{i}$ cannot be 'derived' from $T_{j}$, where $0 \leqslant j<i \leqslant n$. In this case $T_{0}, T_{1}, \ldots T_{n}$ constitutes theoretical progress. Second, there is what may be called 'empirical progress'. $T_{0} \ldots T_{n}$ constitutes empirical progress if, corresponding to the 'approximate derivations' of $T_{n}$ from $T, T_{n-1}$ from $T_{n} \ldots$ and $T_{o}$ from $T_{1}$, we can define approximate versions of these theories, $\mathrm{T}_{0}^{*}, \mathrm{~T}_{\mathrm{n}-1}^{*}, \ldots \mathrm{T}_{0}^{*}$, such that $\mathrm{T} \rightarrow \mathrm{T}_{\mathrm{n}}^{*} \rightarrow \mathrm{T}_{\mathrm{n}-1}^{*} \rightarrow \ldots \mathrm{T}_{0}^{*}$, but $\mathrm{T}_{\mathrm{j}}^{*} \rightarrow \mathrm{T}^{*}{ }_{\mathrm{i}}$ where $0 \leqslant j<i \leqslant n$. (All theories here are assumed to be empirically testable.) The problem is solved. 
(ii) Let us suppose that a theory $\mathrm{T}_{0}$ is succeeded by a theory $\mathrm{T}_{1}$ in the kind of way discussed in (i). Suppose that $T_{0}$ and $T_{1}$ postulate unobservable entities $E_{0}$ and $E_{1}$ respectively. Suppose further that $T_{0}$ is incompatible with $T_{1}$ in such a way that if $T_{1}$ is true (and $E_{1}$ exist) then $E_{0}$ do not exist. In these circumstances, how can $\mathrm{T}_{0}$ be said to provide us with knowledge about unobservable entities, assuming that $T_{1}$ is nearly enough true, as it were, to ensure that $E_{0}$ do not exist?

We assume that, as we move from $\mathrm{T}_{0}$ to $\mathrm{T}_{0}^{*}$ (as defined above) so we move from a theory $\left(\mathrm{T}_{0}\right)$ which postulates precise entities with precise properties, $\mathrm{E}_{0}$, to a theory ( $\mathrm{T}_{0}^{*}$ ) which postulates imprecise entities with imprecise properties, $\mathrm{E}_{0}^{*}$. As an example of this distinction between precise and imprecise entities, consider the following two versions of Newtonian theory interpreted to be about unobservable point-particles interacting by means of gravitation.

NT: Point-particles have precise Newtonian gravitational charge in the sense that the particles obey precisely $\mathrm{F}=\mathrm{Gm}_{1} \mathrm{~m}_{2} / \mathrm{d}^{2}$.

NT*: Point-particles have imprecise gravitational charge in the sense that the particles obey the imprecise law $\mathrm{F}=\mathrm{Gm}_{1} \mathrm{~m}_{2} / \mathrm{d}^{\mathrm{r}}$ with $\mathrm{r}$ some number between $1 \cdot 5$ and $2 \cdot 5$.

Here the point-particles of NT are precise unobservable entities, whereas those of $\mathrm{NT}^{*}$ are imprecise, vague or approximate.

Granted the truth of $T_{1}$, and the existence of $E_{1}$, we have also that $T_{0}^{*}$ is true and that $\mathrm{E}_{0}^{*}$ exist. We can identify $\mathrm{E}_{0}^{*}$ with entities $\mathrm{E}_{1}$ in some special state.

Suppose, for example, that $\mathrm{T}_{0}$ is atomic theory with atoms interpreted to be 'corpuscles' - entities that are indestructible and without internal parts; suppose further that $T_{1}$ is the Rutherford-Bohr theory of atoms. Here $T_{0}$ and $T_{1}$ are incompatible. If atoms $\mathrm{E}_{1}$ (in the sense of $\mathrm{T}_{1}$ ) exist, then corpuscles $\mathrm{E}_{0}$ (in the sense of $\mathrm{T}_{0}$ ) do not exist. Given $\mathrm{T}_{0}$, we can however define $\mathrm{T}_{0}^{*}$, which asserts merely that corpuscles behave as if indestructible and without internal parts. We can 'derive' $\mathrm{T}_{0}^{*}$ from $\mathrm{T}_{1}$ by restricting the domain of $\mathrm{T}_{1}$ to systems of atoms interacting at sufficiently low energies for the atoms to remain in the ground states. In this domain the atoms $\mathrm{E}_{1}$ of $\mathrm{T}_{1}$ are (identical to) the (imprecise) corpuscles $\mathrm{E}_{0}^{*}$ of $\mathrm{T}_{0}^{*}$.

All this provides a basis for claiming that existing empirically successful physical theories ostensibly about unobservable entities do genuinely provide us with theoretical knowledge about unobservable entities even though these theories are, strictly speaking, false.

5. According to physicalism, as understood here, a part of what exists, the invariant, $\mathrm{U}$, determines how another part, the variable, $\mathrm{V}$, does vary or change. But - quite generally - how can what exists detemine change? Standard scientific realism (as defended, for example, by Karl Popper [1963], Ch. 3; J. J. C. Smart [1963], Grover Maxwell [1962], and most of van Fraassen's 
critics: see Churchland and Hooker [1985]) fails to answer this question. For an answer we need to turn to conjectural essentialism.

According to conjectural essentialism it is legitimate to interpret (appropriate) physical theories as attributing necessitating properties to postulated physical entities - properties in virtue of which the entities must, of necessity, obey the laws of the theory. Conjectural essentialism thus goes one substantial step further than standard scientific realism. Whereas standard scientific realism interprets (appropriate) physical theories so that they assert the real physical existence of entities they ostensibly postulate, conjectural essentialism in addition interprets such theories so that they attribute necessitating properties to the entities - properties which, if they exist, require, as a matter of logic, that the entities obey the laws of the theory. Theories interpreted in this essentialistic way postulate the existence of that which explains why the laws of the theory are obeyed; for theories interpreted realistically but not essentialistically, it remains a mystery as to why the entities of the theory should obey the theory's laws. ${ }^{2}$

Philosophers have, unfortunately, made a great mystery out of something that is actually quite simple, and ought readily to be understood by everyone, namely: the idea of a property determining change. ${ }^{3}$ Almost all-perhaps allphysical properties have this character. That is, they are dispositional or necessitating properties which determine how things change. This is true of such common-sense or macro physical properties as rigid, elastic, solid, sticky, heavy, rough, opaque, transparent, inflammable. It is also true of such theoretical properties as electric charge, electromagnetic intensity and direction (of the electromagnetic field), inertial rest mass, spin, gravitational charge. In all such cases, if an object has the property (rigidity, charge or

${ }^{2}$ Conjectural scientific essentialism has one further striking advantage over scientific realism of the type defended by Smart. As a result of making clear what kind of feature or property of things it is that fundamental physics is concerned to specify, conjectural essentialism also makes clear that there can be other kinds of features or properties, above all experiential features-which physics would not describe even though they exist. Conjectural essentialism makes clear, in other words, that physics is only concerned with a highly selected aspect of all that which exists. Conjectural essentialism transforms the two-aspect theory of the mind/body relationship into a thoroughly defensible position about which one may claim that it solves (the conceptual part of) the mind/body problem (see Maxwell [1966, 1968a, 1968b, 1976] and [1984], Ch. 10). In one sense, then, conjectural essentialism is a much stronger doctrine than Smart's scientific realism, in that it interprets (appropriate) fundamental physical theories as attributing necessitating properties to postulated physical entities; in another sense, however, it is a weaker (and much more plausible and illuminating) doctrine in that it allows for the existence of non-physical experiential features of things (colours and sounds as we experience them, and mental features of brain processes), whereas Smart's realism does not.

3 An exception is Ian Thompson who has recently defended an essentialistic interpretation of physics similar to the view being advocated here: see Thompson [1988]. 
whatever), then, in such and such circumstances, of necessity, the object participates in change (or resistance to change) in such and such a way. To say of an object that it is inflammable is to say that it is such that if it is exposed to a naked flame then, of necessity, it burns. There is nothing mysterious about the notion of necessity that is involved here. It is straightforward analytic necessity. In other words, it is built into the meaning of 'inflammability' that inflammable objects burn when exposed to naked flames. Just as 'If $\mathrm{X}$ is a bachelor then X is unmarried' is true analytically (and therefore necessarily) in virtue of the meaning of 'bachelor', so too, 'If X is inflammable and exposed to a naked flame then X burns' is true analytically (and therefore necessarily) in virtue of the meaning of 'inflammable'.

We cannot know for certain that any object, $\mathrm{X}$, is inflammable in this sense, even if we have established that $X$ does always as a matter of fact burn when exposed to naked flames. But this does not make this notion of 'inflammable' meaningless. (Only those who believe in the verification principle of logical positivism could believe this.)

Two notions of 'inflammable' can be distinguished, namely: inflammable ${ }_{1}$ which means 'in fact burns whenever exposed to a naked flame', and inflammable ${ }_{2}$ which means 'of necessity burns whenever exposed to a naked flame'. An object X may be inflammable ${ }_{1}$ and yet not inflammable ${ }_{2}$. It might just so happen that $X$ burns on all those occasions when it is exposed to a naked flame but would not have so burnt on a number of other possible occasions; it might be simply that $\mathrm{X}$ is never exposed to a naked flame, the condition 'burning whenever exposed to a naked flame' being satisfied vacuously. In either case, $X$ is inflammable ${ }_{1}$ but not inflammable 2 . $X$ would burn if it were exposed to a naked flame' may be interpreted in such a way that it is true if and only if $X$ is inflammable ${ }_{2}$ ( $X$ being inflammable ${ }_{1}$ being insufficient). In what follows, 'inflammable' is to be understood to mean 'inflammable ${ }_{2}$ '.

The thesis that $\mathrm{X}$ is inflammable provides only a very inadequate explanation of the fact that $X$ burns when exposed to a naked flame. But this does not mean, as some have thought, that necessitating properties such as inflammability have no proper place in science. The explanation is inadequate because it is extremely restricted in scope. A more adequate explanation would refer to necessitating properties much more widely possessed by physical entities. Such an explanation would link combustion to other phenomena; it would explain why some materials are inflammable, others not, and why objects become and cease to be inflammable in the circumstances in which they do. All this is achieved, at least in principle, by quantum theory applied to chemistry: as a result of attributing a few precise theoretical necessitating properties, such as charge and spin, to electrons and nuclei, it is possible, in principle, to predict and explain a wide range of physical and chemical properties of substances. Inflammability turns out to be the possession of a molecular structure which is such that, at a sufficiently high temperature, in the presence of sufficient 
oxygen, oxidization proceeds rapidly and in a self-sustaining way, with the emission of gas, heat and light. A highly theoretical quantum mechanical description of combustion provides a very much better explanation because, as a result of attributing very few necessitating properties to very few different kinds of entities, a vast range of apparently diverse phenomena can in principle be predicted, including phenomena associated with inflammability. In order to get good explanations, in other words, we do not need to get rid of references to necessitating properties: we need rather to discover necessitating properties that determine a wide range of phenomena, of kinds of change.

We may regard the quantum mechanical explanation as providing the underlying mechanism involved in combustion - as telling us what inflammability really is, in our world. It is important to appreciate, however, that in attributing the necessitating property of inflammability to something, we do not thereby require there to be some such underlying mechanism, some such theoretical explanation. It is logically possible that the world is such that all the diverse observational necessitating properties that there appear to be in the world around us do really exist, and yet there exists no mechanism underlying these properties-nothing in terms of which a theoretical explanation of the properties can be couched. In calling something inflammable we leave open, but do not require, that inflammability can be explained, or explained away, in terms of more widely possessed properties. Necessitating properties exist in possible worlds that are incomprehensible, in other words, as well as in physicalistically comprehensible possible worlds.

This point is important because if we make possessing a necessitating property dependent on the possibility of a theoretical explanation (or, ultimately, dependent on physicalism being true), then we cannot interpret fundamental physical theories (or versions of physicalism) essentialistically, as attributing essentialistic properties to postulated entities. From the standpoint of theoretical physics, the entire raison d'etre for having the notion of necessitating property disappears.

Any fundamental physical theory amenable to being interpreted realistically can also be interpreted essentialistically, as attributing necessitating properties to postulated entities which require, as a matter of logic, that the entities obey the laws of the theory. Newtonian theory interpreted realistically as a theory about point-particles with inertial mass subject to Newton's laws asserts, when interpreted essentialistically: (1) Everything is made up of pointparticles with invariant inertial mass $m$ and gravitational charge $\mathrm{g}$, with $\mathrm{m}=\mathrm{g}$. Here (2) $\mathrm{F}=\mathrm{ma}$ is true analytically, in virtue of the meaning of 'inertial mass' and 'force'; and likewise (3) $\mathrm{F}=\mathrm{Gg}_{1} \mathrm{~g}_{2} / \mathrm{d}^{2}$ is true analytically, in virtue of the meaning of 'gravitational charge'. This does not make Newtonian theory itself analytic, for (1) is a massively contingent assertion (which is in fact false given general relativity or quantum theory). We have in fact any number of 
different interpretations of Newtonian theory, from the maximally essentialistic at one extreme, to the more and more anti-essentialistic at the other extreme, the meaning of 'force', 'inertial mass' and 'gravitational charge' becoming progressively more and more vague, presupposing the truth of laws that are more and more vague, as we move from the essentialistic to the antiessentialistic. Thus if the meaning of 'gravitational charge ${ }_{1}$ ' is such that ' $\mathrm{F}=\mathrm{Gg}_{1} \mathrm{~g}_{2} / \mathrm{d}^{2}$ ' is true analytically, the meaning of 'gravitational charge ${ }_{5}$ ' might be such that no more than 'objects with gravitational charge ${ }_{5}$ tend to move towards each other in some way that is proportional to their mass' is true analytically. Any such more or less essentialistic theory is only true if the entities of the theory really exist with all the necessitating properties which the theory attributes to them.

In a deterministic universe governed by a deterministic physical theory, $\mathrm{T}$, which is true even when interpreted in a maximally essentialist manner, what exists at one instant determines necessarily what exists at subsequent instants. Let $S_{0}$ and $S_{1}$ be complete, precise specifications of the state of the universe at times $t_{0}$ and $t_{1}$ with $t_{1}>t_{0}$. Then as long as the universe is such that $T$ is only true when interpreted non-essentialistically, $S_{0}$ does not imply $S_{1}$. It is rather $S_{0}$ plus $\mathrm{T}$ that implies $\mathrm{S}_{1}$. If, however, the universe is such that $\mathrm{T}$ is true even when interpreted in a maximally essentialist way, all the laws of $T$ are analytic. $S_{0}$, in order to give a complete specification of what exists at time $t_{0}$, must attribute necessitating properties possessed by entities at $t_{0}$ which presuppose that the entities obey $\mathrm{T}$. In other words, $\mathrm{S}_{0}$ must include $\mathrm{T}$ implicitly in specifying what exists at $t_{0}$. Thus in this case $S_{0}$ on its own implies $S_{1}$. There are logically necessary connections between successive states of the universe $S_{0}$ and $S_{1}$, in the sense that what exists at $t_{0}$ is such that any set of propositions which correctly and completely describes it logically implies propositions which describe subsequent states of the universe. If we try to weaken $S_{0}$ so that it becomes $S_{0}^{*}$ let us say, with $S_{0} \rightarrow S_{0}^{*}$ but $S_{0}^{*} \nrightarrow S_{0}$, and $S_{0}^{*} \nrightarrow S_{1}$, then an aspect of what exists at $t_{0}$, described by $S_{0}$, is not described by $S_{0}^{*}$. It is thus what exists at $t_{0}$ that necessarily determines the subsequent state of affairs at time $t_{1}$.

This deterministic notion of necessitating property can be generalized to form a probabilistic notion of necessitating property: for a discussion of this notion, its relevance to interpreting probabilistic quantum theory, and how it differs from Popper's closely related propensity notion, see Maxwell [1976a, 1985, 1988].

Most philosophers, even today, reject the whole idea that necessitating properties of the above type might really exist. They do this, essentially, because they take it for granted that David Hume, long ago in 1738, showed decisively that the whole idea of a necessitating property, discussed above, does not make sense (Hume [1959]). It has not been noticed just how crudely defective Hume's arguments are. The entire argument is based in an essential 
way on a phenomenalistic criterion of meaning: all meaningful ideas must be analysable into sense data terms-or, as Hume puts it, 'all our ideas are copied from our impressions' (Hume [1959], p. 76). Being unable to trace the notion of necessitating property or necessary connection, as conceived of here, to sense impressions, Hume concludes that there are no such notions, the whole idea of there being necessary connections between successive events in Nature being meaningless. But phenomenalism quite generally, including Hume's version, is wholly untenable - as everyone today agrees. This demolishes Hume's entire argument.

Few philosophers even today seem to appreciate just how grossly defective Hume's arguments are, even though I sought to point this out over twenty years ago now (see Maxwell [1968a]. ${ }^{4}$

Once phenomenalism is rejected, all that remains of Hume's argument is something like the following: just as whatever object happens to exist at one place cannot determine necessarily what exists at another place, so too what object (instantaneous event or state of affairs) exists at one time cannot determine necessarily what exists at another time.

There are two ways in which one may seek to demolish this argument. The first challenges the validity of the argument: time, it may be argued, need not be sufficiently like space for the argument from space to time to be valid. The second leaves the validity of the argument unquestioned and challenges the premise. Necessary connections between different parts of space are possible. And hence, too, it is possible to have necessary connections between different times.

In earlier publications I have developed the first argument. Here I sketch the second argument. It is a rather more powerful argument in that it transforms an argument against the possible existence of necessary connections between successive events into an argument for the possible existence of such connections.

The argument for the possible existence of necessary connections between distinct places (spatial as opposed to temporal necessary connections) proceeds as follows. If we have two distinct objects each confined to one of two distinct spatial regions, $r_{1}$ and $r_{2}$, then it may well be true that what exists at $r_{1}$ cannot necessarily determine what exists at $r_{2}$. But suppose we have one object occupying both $r_{1}$ and $r_{2}$. And suppose that this object is such that (i) it cannot overlap spatially with any other object, and (ii) the nature of the whole object is

4 This is perhaps an overstatement. Since my 1968 paper, a few philosophers have defended the anti-Humean idea that laws of nature are necessary: see Dretske [1977], Tooley [1977] and Armstrong [1978] and [1983]. These attempts have been severely criticized by van Fraassen [1989]. Note that none of van Fraassen's arguments against the possibility that laws of nature are necessary applies to the position defended in my 1968 paper-or to the conjectural essentialism defended here. Van Fraassen completely ignores my 1968 paper. 
determined necessarily by any spatial part of the object, however small. In this case what exists in $r_{1}$ would necessarily determine what exists in $r_{2}$.

There are, here, two kinds of objects to consider: spatially contingent objects and spatially necessary objects. A spatially contingent object is an object which can be specified precisely and completely by a specification of what exists at each spatial point the object occupies, the omission of a specification of what exists at any one occupied point being sufficient to render the specification of the whole object incomplete. Spatially necessary objects are objects for which this is not true. One kind of spatially necessary object is an any-point-selfdetermining object. This object is such that what exists at any occupied point is such that it necessarily determines what exists at all other occupied points. The object, $\mathrm{O}$, is such that at each occupied point $(\mathrm{x})$ there is a particular value, $\mathrm{P}(\mathrm{x})$, of a necessitating property, $\mathrm{P}$, which determines necessarily that $\mathrm{O}$ and $\mathrm{P}$ exist throughout all occupied points, and determines necessarily what $\mathrm{P}(\mathrm{x})$ will be at each $(\mathrm{x})$.

The Newtonian gravitationally charged point-particle, conceived of in a maximally essentialistic way, is a spatially necessary object (ignoring that it fails to exclude other objects). This object, via its gravitational charge, occcupies all of space (since the gravitational force created by the charge has a value greater than zero throughout all of space). This entire object is, however, determined necessarily by what exists at the point occupied by the pointparticle - the value of the Newtonian gravitational charge, or inertial mass, at that point. Any specification of this essentialistic Newtonian point-particle which satisfies the requirements for spatial contingency must be incomplete because it cannot specify the spatially necessitating property of gravitational charge which the point-particle possesses at the point it occupies.

A variety of different kinds of spatially necessary objects are conceivable. There is the object that is determined necessarily by what exists in some particular proper part of the space it occupies. There is the object that is determined necessarily by what exists within any volume, $\mathrm{dV}$, of occupied space, however small. There is the object that is determined necessarily by what exists at one unique point. And there is the object that is determined necessarily by what exists at any $\mathrm{N}$ points-with $\mathrm{N}=1$ as a special case. The important point here, however, is that the mere possibility or conceivability of any of these spatially necessary objects suffices to establish that it is possible that what exists at one place does necessarily determine what exists at another place. Not only does the above remnant of Hume's argument fail to establish that it is impossible for temporally necessary connections to exist; it actually establishes that it is possible for temporally necessary connections to exist (granted the validity of arguing from space to time).

One great advantage that this argument has over my earlier argument (Maxwell [1968a], pp. 153-7) is that it renders the existence of necessary 
connections entirely compatible with special relativity. The earlier argument, in requiring there to be an essential distinction between space and time, makes the existence of necessary connections problematic granted special or general relativity. No such difficulty arises in connection with the new argument.

The two arguments can, however, be brought together to form one coherent argument, which may be outlined as follows. It is possible that physical space is such that it renders spatial necessary connections impossible. But even if space is like this, it is also possible that time is sufficiently different from space so that temporal necessary connections are possible. There are grounds, however, for holding that spatial necessary connections are only impossible in some physical spaces but not all. In order to exclude spatial necessary connections - or spatially necessary objects-space needs itself to possess a necessitating property: one which necessarily excludes spatially necessary objects. Strikingly enough, such a necessitating property, in order to exclude spatially necessary objects in space, must be such that it turns space itself into a spatially necessary object. In other words, in order to deny the possibility of spatially necessary objects we need to assert that physical space itself is a spatially necessary object. It is quite possible that space is not this kind of spatially necessary object (which excludes the existence of all other spatially necessary objects). If this is the case, then spatially necessary objects (and connections) become entirely possible (as indicated above). In this case we do not at all need to draw a distinction between space and time in order to make possible temporal necessary connections.

Essentialistic physics is able to explain why regularities exist in Nature. Regularities are the outcome, the necessary consequence, of physical entities possessing appropriate necessitating properties. But anti-essentialistic physics, by contrast, can provide no such explanation. That there should be precise, universal regularities in Nature must remain an absolute mystery, a persistent miracle. It cannot help at all to 'explain' regularities that are restricted in scope by demonstrating that they are consequences of much more universal regularities. Such 'explanations' only deepen the mystery -in that the more universal a mere regularity is, so the more inexplicable it becomes. It is, in short, absurd to suppose that there could be precise, universal regularities in Nature, and yet nothing in existence which is responsible for such regularities. Yet it is just this absurdity to which anti-essentialistic physics commits itself (assuming that no other agent, such as God, is called upon to be responsible for maintaining the lawfulness of Nature).

The outcome is clear: physics must be interpreted essentialistically. Of course, if Hume were right, and no meaning could be given to the notion of necessitating property, this whole line of reasoning would collapse. We have seen, however, that Hume is wrong. Given the possibility of necessitating 
properties, it becomes absurd not to commit physics to the essentialistic task of discovering the nature of that necessitating property, invariantly present everywhere, which is responsible for determining how things change.

Physicists, as well as philosophers, have rejected conjectural essentialism for bad reasons. There are at least four. They stem from (i) anti-Aristotelianism, (ii) belief in God, (iii) respect for Newton, and (iv) acceptance of standard empiricism. I take these in turn. (i) In creating modern science, Galileo and others appreciated that it was necessary to reject Aristotelianism and put in its stead a mechanistic, mathematical or atomistic view of nature-i.e. some version of physicalism. This meant in particular rejecting the 'occult' properties of Aristotelianism and the pseudo explanations they provide. But occult and necessitating properties seem superficially to be very similar. In vehemently opposing the former, scientists created a psychological barrier to the idea that science might need to refer to the latter-essentialism being in this way excluded from consideration. (ii) Most of those who created modern science believed in the existence of God. Belief in God even played an important role in the creation of science: it inspired belief in the order and knowability of the universe. But belief in God renders belief in necessitating properties redundant: one can believe that lawfulness exists because God wills it. As a result natural philosophers were able to develop physics as a science of natural laws or regularities without there being any scientific explanation as to why lawfulness exists - the explanation lying (so it was believed) beyond science in religion. Later, when science became dissociated from its Christian, theological context, the anti-essentialist mode of thought, which no longer made sense had, within science, hardened into an unquestionable dogma. (iii) Newton, notoriously, vehemently rejected any essentialistic interpretation of his law of gravitation. For Newton, this law described the actual motions of objects, but did not explain these motions, and did not assert the existence of a real physical force of gravitation acting at a distance. Not surprisingly, given the immense prestige of Newton, this anti-essentialistic attitude became enshrined in subsequent physics. (iv) Most physicists after Newton have taken some form of standard empiricism for granted. Standard empiricism requires that science be sharply dissociated from philosophy and metaphysics. But in order merely to distinguish essentialism and anti-essentialism, and in order to develop and assess arguments in support of the former and against the latter, it is necessary to do philosophy and metaphysics. Standard empiricism thus excludes from physics just that which needs to be brought into physics if anti-essentialism is to be reconsidered.

Conjectural essentialism strengthens scientific realism against its critics in a number of ways.

First, it is required in order to make sense of physicalism, as I have formulated it in this paper. Deny essentialism as a possibility, and it becomes impossible to make sense of the idea that something exists which determines 
necessarily the lawfulness of nature.$^{5}$ Physicalism degenerates into a doctrine which asserts no more than that unified lawfulness reigns supreme without there being anything in existence which is, in any sense, responsible for this lawfulness. Such an emasculated version of physicalism is difficult to take seriously. The basis for aim-oriented empiricism as I have expounded it above is sabotaged.

Second, without conjectural essentialism there is no clear understanding of how physical properties of entities are related to laws obeyed by the entities. This makes it possible for physicists to try to understand new laws and theories in terms of old and grossly inappropriate entities and properties-even to the extent of finding the new laws and theories unintelligible if they cannot be understood in this (grossly misconceived) way. Just this pattern of confusion is to be found again and again in the history of physics. Thus Newton's law of gravitation is found to be inexplicable because it is an action-at-a-distance law, and therefore cannot be understood in terms of action-by-contact-in terms of corpuscles, in other words. Only with Boscovich do we get the creation of an idea for physical entities and properties appropriate to Newton's law-namely the Boscovichean point-particle. Again, Maxwell's theory of the electromagnetic field is found to be inexplicable because it cannot be understood in terms of the aether, i.e. in materialistic terms, that is, in terms of the point-particle or the corpuscle. Not till Einstein (leaving Faraday on one side) do we get a clear recognition that the field is a new kind of physical entity in its own right, with new kinds of properties. Yet again, fundamentally probabilistic quantum theory is found to be inexplicable because it cannot be understood in terms of (grossly inappropriate) deterministic, classical physical entities- the field, the point-particle. We still do not have a clear recognition that in order to

${ }^{5}$ What it is that physicalism asserts (as understood here) can perhaps be further clarified by considering the following example of a physicalistic universe. Suppose that all that exists is the classical electromagnetic field. In this case, the nature of the unified, invariant something responsible for all change, is specified by the following essentialistically interpreted postulates:

$$
\begin{aligned}
& \text { (1) } \nabla \cdot E=0 ; \\
& \text { (2) } \nabla \cdot B=0 ; \\
& \text { (3) } \nabla \times E=-\frac{1}{C} \frac{\partial B}{\partial t} \text {; } \\
& \text { (4) } \nabla \times B=\frac{1}{C} \frac{\partial E}{\partial t}
\end{aligned}
$$

(where $\mathrm{E}$ and $\mathrm{B}$ are the electric and magnetic fields respectively, and $\mathrm{c}$ is the velocity of light). These postulates are analytic; they specify how $\mathrm{E}$ and $\mathrm{B}$ must change, from place to place and time to time, if the invariant something, the electromagnetic field, is to exist everywhere, at all places and times, without changing. The fragment of Maxwellian electrodynamics that we are considering, in order to be a contingent theory, needs an additional, non-analytic existence claim in the form of a fifth postulate: (5) The electromagnetic field, as characterized in (1) to (4), exists everywhere. (If it exists everywhere at any given instant then it necessarily exists everywhere at all subsequent instants.) We have here, then, an example of a universe that is physicalistically comprehensible. 
understand quantum theory we need to interpret it in terms of a new kind of probabilistic entity with probabilistic properties. Much effort has been wasted on attempting what ought never to have been attempted in the first place, namely: interpreting new theories in terms of inappropriate entities that relate to earlier, different theories. From the standpoint of conjectural essentialism it is dazzlingly clear that all this is wasted effort. To think of entities and their properties is to think of the laws the entities obey. Developing fundamentally new theories trivially requires the corresponding development of fundamentally new ideas concerning entities and properties. This simple point has farreaching consequences for the whole way one conceives of, and tries to solve, contemporary interpretative problems of quantum theory (see Maxwell [1988]). ${ }^{6}$

The grossly misguided endeavour of trying to understand new theories in terms of old entities gives way, when inevitably it fails, to anti-realism. This is to be found in connection with Newtonian theory, classical electromagnetism and quantum theory. It is one of the main sources of anti-realism. What conjectural essentialism makes clear is that the source of the trouble is not realism at all, but rather grossly misguided realism, anti-essentialistic realism which dissociates properties from laws.

6. How might van Fraassen reply to the argument of this paper? I imagine that his main line of reply would be to object to my claim that in order to solve the problem of induction we need to reject standard empiricism and adopt aimoriented empiricism instead. I do not think that this objection can succeed: the argument against standard empiricism seems to me to be decisive. There is, however, another kind of reply which van Fraassen might make: even if, in order to solve the problem of induction, we do require some version of aimoriented empiricism, this version need not imply scientific realism. For in order to solve the problem of induction all we require is that science presupposes that

${ }^{6}$ Quantum theory, given its orthodox interpretation (OQT), has instrumentalism built into it, in that the theory is about the results of performing measurements on systems, and not about quantum systems per se. This is sometimes thought to provide grounds for accepting instrumentalism and rejecting scientific realism. Actually, the situation is all the other way round. As I have argued elsewhere (Maxwell [1972, 1976a, 1982, 1988]) OQT is severely defective, in ways which even instrumentalists or constructive empiricists ought to acknowledge, just because OQT cannot be interpreted realistically. OQT is (1) imprecise, (2) severely ad hoc, and (3) restricted in scope, as a result of its lack of realism. (OQT is even more obviously defective when viewed from the perspective of aim-oriented empiricism.) Furthermore, any fundamental physical theory which has instrumentalism built into it in this manner will, as a result, have these defects. There is here, then, a strong additional argument in support of scientific realism. We do not even have grounds, let it be noted, for holding that it is supremely difficult to modify OQT so that it becomes a fully realistic theory, with its own distinctive quantum ontology; what has blocked efforts in this direction (until recently) has been, not so much the difficulty of the physics, as the influence of bad instrumentalistic philosophy, promulgated most notably by Bohr, which has denied the existence of the problem. 
the universe is such that it behaves, from an observational standpoint, as if some version of physicalism is true. More precisely, all we require is that science presupposes that the universe is such that some as-yet-to-bediscovered perfectly unified, explanatory theory $\mathrm{T}$ is, for all phenomena, empirically adequate. Science need only presuppose constructive physicalism (as we may call this doctrine); and constructive physicalism clearly does not imply or require scientific realism.

The basic idea of the aim-oriented empiricist solution to the problem of induction outlined above is that we are rationally obliged to conjecture, within the context of science, that the universe is comprehensible in some way or other; that is, that there is something which is, in some sense, responsible for everything that happens. The precise way in which the universe is comprehensible is something we have to discover by comparing the relative empirical successes and failures of rival research programmes based on rival ideas as to how the universe may be comprehensible. One general possibility is physicalism. Constructive empiricism is, however, not a contender. A universe which only behaves, from an observational standpoint, as if it is physicalistically comprehensible, there being no largely unobservable entity, U, invariantly present everywhere, determining necessarily the way in which that which changes does change is, from the standpoint of what exists, an incomprehensible universe. The empirically adequate theory, $\mathrm{T}^{*}$ (where $\mathrm{T}^{*}$ is $\mathrm{T}$ interpreted to be only about what is observable), would be a grossly ad hoc, aberrant theory. There could be no raison d'être for preferring T* to infinitely many rival theories, all just as aberrant as $\mathrm{T}^{*}$, and all just as compatible with all available evidence. Allowing science to presuppose only that some version of constructive physicalism is true does not, in short, suffice to solve the problem of induction. The above objection must be rejected.

Department of History and Philosophy of Science University College London

\section{REFERENCES}

Armstrong, D. [1978]: A Theory of Universals, Cambridge University Press. Armstrong, D. [1983]: What is a Law of Nature?, Cambridge University Press.

Churchland, P. M. AND Hooker, C. A. (eds.) [1985]: Images of Science, University of Chicago Press.

DretSKe, F. [1977]: 'Laws of Nature', Philosophy of Science, 44, pp. 248-68.

Hume, D. [1959]: A Treatise of Human Nature, Dent.

KRIPKE, S. [1980]: Naming and Necessity, Basil Blackwell.

Laudan, L. [1980]: 'A Confutation of Convergent Realism', Philosophy of Science, 48, pp. $19-48$. 
Maxwell, G. [1962]: 'The Ontological Status of Theoretical Entities', in H. Feigl and G. Maxwell (eds.), Minnesota Studies in the Philosophy of Science, 3, University of Minnesota Press, pp. 3-27.

Maxwell, N. [1966]: 'Physics and Common Sense', British Journal for the Philosophy of Science, 16, pp. 295-311.

Maxwell, N. [1968a]: 'Can there be Necessary Connections between Successive Events?', British Journal for the Philosophy of Science, 19, pp. 1-25.

MAxwell, N. [1968b]: 'Understanding Sensations', Australasian Journal of Philosophy, 46, pp. 127-46.

Maxwell, N. [1972]: 'A New Look at the Quantum Mechanical Problem of Measurement', American Journal of Physics, 40, pp. 1431-5.

Maxwell, N. [1976a]: 'Towards a Micro Realistic Version of Quantum Mechanics', Foundations of Physics, 6, pp. 275-92 and 661-76.

MAXWELL, N. [1976b]: What's Wrong With Science? Bran's Head Books.

Maxwell, N. [1982]: 'Instead of Particles and Fields', Foundations of Physics, 12, pp. 607-31.

MAXWELl, N. [1984]: From Knowledge to Wisdom: A Revolution in the Aims and Methods of Science, Basil Blackwell.

Maxwell, N. [1985]: 'Are Probabilism and Special Relativity Incompatible?', Philosophy of Science, 52, pp. 23-44.

Maxwell, N. [1988]: 'Quantum Propensiton Theory: A Testable Resolution of the Wave/Particle Dilemma', British Journal for the Philosophy of Science, 39, pp. 1-50.

Miller, D. [1974]: 'Popper's Qualitative Theory of Verisimilitude', British Journal for the Philosophy of Science, 25, pp. 166-77.

Newton-Smith, W. [1981]: The Rationality of Science, Routledge.

PopPER, K. R. [1963]: Conjectures and Refutations, Routledge \& Kegan Paul.

SMArt, J. J. C. [1963]: Philosophy and Scientific Realism, Routledge \& Kegan Paul.

Thompson, I. [1988]: 'Real Dispositions in the Physical World', British Journal for the Philosophy of Science, 39, pp. 67-79.

Tichý, P. [1974]: 'On Popper's Definition of Verisimilitude, British Journal for the Philosophy of Science, 25, pp. 155-60.

Tooley, M. [1977]: ‘The Nature of Laws', Canadian Journal of Philosophy, VIII, pp. 66798.

Van FraAssen, B. C. [1980]: The Scientific Image, Clarendon Press.

VAN FraAssen, B. C. [1985]: 'Empiricism in the Philosophy of Science', in P. M. Churchland and C. A. Hooker (eds.) [1985].

Van FraAssen, B. C. [1989]: Laws and Symmetry, Clarendon Press. 